\title{
Anion/Cation Layers at Electrified Interfaces: A Comprehensive STM, XRD and XPS Case Study
}

\author{
Duc T. Pham a, Hubert Keller ${ }^{b}$, Stephan Breuera, Sascha Huemanna, Nguyen T.N. Haiª, Caroline \\ Zoerlein ${ }^{\mathrm{a}}$, Klaus Wandelta, and Peter Broekmann ${ }^{\star \mathrm{ac}}$
}

\begin{abstract}
Charged organic adsorbates play an important role in a number of electrochemical reactions, e.g. as additives for metal plating relevant for device fabrication in the semiconductor industry. Fundamental investigations are mandatory in order to acquire profound knowledge of the structural and electronic properties of these layers parallel and perpendicular to the surface, and to finally achieve a deeper mechanistic understanding of the kinetics of involved charge transfer reactions taking place at these complex metal/organic/electrolyte interfaces. A key structural motif of these interfaces consists in 'paired' (inorganic)anion/(organic)cation layers that can have an enormous stability even during an ongoing charge transfer reaction. In this contribution we present and discuss a selected case study on the co-adsorption of halide anions and cationic organic molecules that exhibit a pronounced redox activity. It will be demonstrated that their phase behavior at the interface crucially depends on both their particular redox-state and the surface concentration of the halide counter ions. The subtle balance between adsorbate-adsorbate and adsorbate-substrate interaction of the poly-cationic organic layer can be carefully controlled by potential dependent anion adsorption and desorption processes through the organic layer. This process can be followed by in situ high-resolution scanning tunnelling microscopy, while additional information about the structural and chemical state of the respective phase is obtained from in situ X-ray diffraction and ex situ photoelectron spectroscopy.
\end{abstract}

Keywords: In situ STM · SXPS · SXRD · Copper electrochemistry · Electron transfer reaction · Surface phase transition

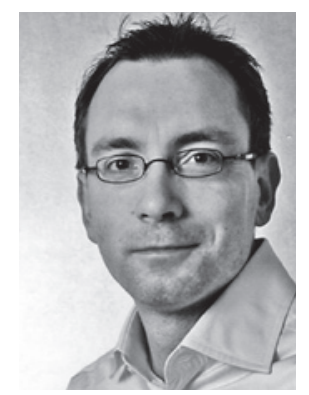

Peter Broekmann studied chemistry at the University of Bonn, where he gained $\mathrm{PhD}$ in physical chemistry in 2000. After a postdoctoral stay in the group of Prof. Bene Poelsema at the University of Twente (Netherlands) he started a habilitation project at the Institute of Physical Chemistry in Bonn. Since October 2007 he is member of the BASF copper plating team. In October 2008 he accepted a lecturer position for interfacial electrochemistry at the University of Bern. His research is focused on copper electrochemistry utilizing in situ scanning probe techniques, in situ X-ray diffraction and ex situ photoelectron spectroscopy.

${ }^{*}$ Correspondence: Dr. P. Broekmann ${ }^{\text {ac }}$

Tel.: +41316314317

Fax: +41316313994

E-mail: peter.broekmann@iac.unibe.ch

alnstitut für Physikalische und Theoretische Chemie Universität Bonn

Wegelerstr. 12, 53115 Bonn, Deutschland

'Institut für Theoretische und Angewandte Physik

Universität Stuttgart

Pfaffenwaldring 57, 70569 Stuttgart, Deutschland

'Department of Chemistry and Biochemistry

University of Bern

\section{Introduction}

The understanding and modelling of the kinetics and reaction pathways of interfacial charge transfer reactions taking place in an electrochemical environment requires a detailed knowledge not only of the physicochemical properties of the reactants in the bulk solution phase but also of the atomic scale structure of the respective interface. However, for numerous technologically and economically relevant processes such a fundamental understanding of the interfacial structure is lacking. The reason for this was related for a long time to the lack of suitable in situ analytical tools allowing an atomic-scale characterization of these electrified interfaces. Within the last three decades, however, many efforts have been made in particular due to the successful implementation of modern structure-sensitive surface science techniques such as surface X-ray diffraction (SXRD) ${ }^{[1]}$ and scanning tunneling microscopy $(\mathrm{STM})^{[2]}$ into the field of surface electrochemistry.

One of today's most prominent examples for such an enormous discrepancy between the level of knowledge on the one hand and its technological and economical relevance on the other hand concerns the state-of-the-art on-chip wiring of transistors on patterned $\mathrm{Si}$-wafers which is based on an electrochemical copper deposition process, in the literature often referred to as the (Dual) Damascene Process. ${ }^{[3]}$ This process involves the defect-free filling of 'vias' and 'trenches' on the patterned wafer surface with copper. What is typically needed is a so-called superconformal growth mode (superfill) that fills these submicrometer features from the bottom to the top. An electroplating process has been proven as the most cost- and time-saving deposition route with excellent gap-filling capabilities even for sub- $60 \mathrm{~nm}$ inlayed features. ${ }^{[4]}$ The secret of the superfill phenomenon lies in the particular composition of the plating baths. ${ }^{[5]}$ It is the time-dependent interplay of various organic and inorganic additives with both the copper electrode surface itself and with reaction intermediates of the copper deposition (e.g. cuprous ions) in the near-surface regime that finally leads to the desired bottom-up filling. ${ }^{[5-7]}$ Depending on their phenomenological impact on the copper deposition kinetics the plating literature distinguishes so-called accelerator (or catalyst) and suppressor (or inhibitor) additives. ${ }^{[5-7]}$ Typically, short aliphatic compounds which are functionalized by thiol-, disulfide and sulfonic head groups serve as 'accelerators'. ${ }^{[3-5]}$ Also chloride anions which are commonly added in trace amounts to the plating bath are 
known to reveal accelerating capabilities (at least in the absence of other additives) while bromide and iodide are reported to suppress the copper deposition. ${ }^{[8]}$ Watersoluble polymers such as the polyethylene glycol (PEG), polypropylene glycol (PPG) and co-polymers thereof commonly serve as suppressor additives. ${ }^{[6]}$ Their suppressing effect relies on the formation of dense polymer films at the interface during copper deposition thus physically limiting the access of cuprous and cupric ions to the copper surface. A third important class of additives that is commonly present in the copper plating baths is the so-called leveler. While the time-dependent interplay between the accelerator and suppressor regulates the superfill itself the leveler plays a major role only in an advanced stage of the copper deposition process after the successful completion of the gap fill to finally effect a planarization of the copper deposit. Leveler compounds are typically nitrogen-containing, cationic organic additives which might be polymeric [9] or non-polymeric. ${ }^{[10]}$ Common polymeric levelling agents as described in current patents ${ }^{[9]}$ are based on polyethyleneinimes, co-polymers of imidazole and epichlorhydrin and polypyridines which might be quaternized through methylation or benzylation. Common non-polymeric levelling agents are quaternized alkyl amines or quaternized cyclic amines. Often used compounds are imidazole or pyridine derivatives which might be quaternized by alkylation (e.g. benzylation) or arylation (e.g. phenylation) at the nitrogen atoms of the respective heterocycles. ${ }^{[9]}$

Similar to the PEG- and PPG-based suppressors also the leveler forms a dense film on the copper surface acting as an effective diffusion barrier for cuprous and cupric ions. ${ }^{[10]}$ However, in contrast to the PEG- and PPG-based suppressors one important driving force for the leveler film formation relies on electrostatic attraction between specifically adsorbed anions that retain to a large extent their negative charge upon adsorption on the copper surface and the poly-cationic leveler species.[10]

Therefore anion-cation pairing effects at the interface are considered as being crucial in order to explain the differences in the suppressing action modes of PEG/ PPG-based and multi-cationic leveler-type suppressors.

In this review we present a case study of the potential-depended interaction of chloride anions with a multi-cationic organic film (Fig. 1) using selected model systems that allow the use of modern structure-sensitive in situ techniques such as in situ scanning tunneling microscopy (STM) and in situ surface $X$-ray diffraction (XRD). These results are further combined with ex situ surface X-ray photoemission

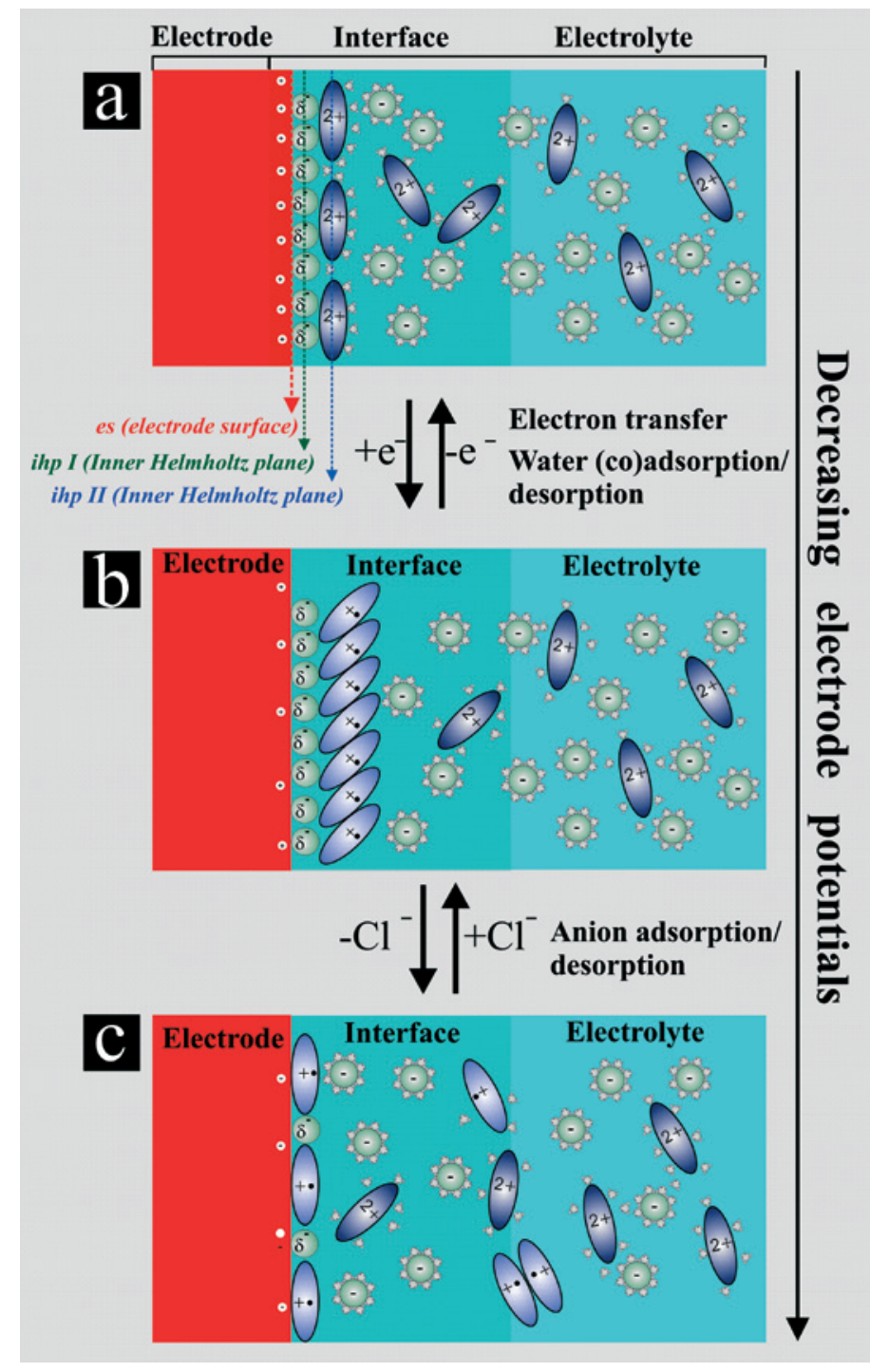

Fig. 1. Schematic representation of the potential-dependent interfacial structure in the presence of redoxactive viologens on the anion-modified electrode surface: a) Adsorption of partially solvated viologens in their di-cationic form on the halide lattice; b) Self-assembly of viologen radical monocations into stacking chains as result of the electron-transfer reaction; c) Partial desorption of anions through the layer of viologen radical monocations.

spectroscopy (SXPS) experiments providing additional information on the chemical composition of the interface.

As model substrate of choice we used a single crystalline $\mathrm{Cu}(100)$ electrode surface exposed to a chloride-containing acidic supporting electrolyte. ${ }^{[11]}$ In order to mimic the formation of a poly-cationic organic film on the chloride modified copper electrode we adsorbed so-called viologens (1,1'-4,4'-disubstituted-bipyridinium molecules) that reveal a pronounced redox activity. ${ }^{[12]}$ This can be used to tune the balance between inter-adsorbate and adsorbate-substrate interactions.

Di-cationic viologen species $\left(\mathrm{V}^{2+}\right)$ can be reversibly transformed into their corresponding radical mono-cations $\mathrm{V}^{\cdot+}$ via a one-electron transfer step according to Eqn. 1:

$\mathrm{V}^{2+}+\mathrm{e}^{-} \leftrightarrow \mathrm{V}^{\bullet+}$

This first electron transfer reaction can be followed by a subsequent dimerization, an oligo- or polymerization of the formed radical mono-cations according to:
$\mathrm{nV}^{\bullet+} \leftrightarrow\left(\mathrm{V}^{\bullet+}\right)_{\mathrm{n}}$

Oligomeric and polymeric reaction products tend to accumulate on the electrode surface ${ }^{[13]}$ (Fig. 1b) while the dimer species often remains water soluble. ${ }^{[12]}$

A second single electron transfer step transforms the radical mono-cation into the fully uncharged viologen species according to Eqn. 3:

$$
\mathrm{V}^{\bullet+}+\mathrm{e}^{-} \leftrightarrow \mathrm{V}^{0}
$$

The latter transition has to be considered as less reversible than the first electron transfer. ${ }^{[12]}$ Note these electron transfer reactions do not necessarily take place at the bare metallic electrode surface. Specifically adsorbed anions from the supporting electrolyte can alter both the geometric and electronic structure of the electrode surface (Fig. 1) and, as a consequence, the entire charge distribution perpendicular to the surface in the near-surface electrolyte regime. ${ }^{[14]}$ The impact of this anion adsorption on the kinetics of electrochemical re- 
actions is well known from the early work of Frumkin. ${ }^{[15]}$ The interfacial behavior becomes even more complex when reactants and/or products of the electron transfer process are adsorbed on the anion-modified electrode surface prior to or during the reaction. Provided the adsorbed anions retain to a large extent their negative charge at the surface the subsequent adsorption of cationic reactants from the electrolyte onto the electrode will be facilitated through electrostatic attraction (Fig. 1a), thus giving rise to the formation of 'paired' anion-cation layers which might remain stable even during a continuing charge transfer reaction. ${ }^{[13]}$ Such an anion-cation layering has recently been reported for the adsorption and subsequent reaction of dibenzyl-viologens (1,1'-dibenzyl-4,4'-bipyridinium molecules, DBV) on a chloride-modified $\mathrm{Cu}(100)$ electrode surface. ${ }^{[13]}$

In this case study we will focus on the diphenyl-viologen (1,1'-diphenyl-4,4'bipyridinium molecules, DPV) which has to be considered as more reactive than the DBV due its conjugated $\pi$-electron spreading over the entire molecule.

\section{Results and Discussion}

\subsection{The Substrate: $\mathrm{Cu}(100)-\mathrm{c}$ $(2 \times 2)-\mathrm{Cl}$}

The interaction of chloride with $\mathrm{Cu}(100)$ is well documented in the literature. ${ }^{[11,13]}$ Chloride anions specifically adsorb on $\mathrm{Cu}(100)$ at high potentials (Fig. 2a) under the formation of an ordered adlayer that can be described by a $\mathrm{c}(2 \times 2)-\mathrm{Cl}$ or $(\sqrt{2} \times \sqrt{2}) \mathrm{R} 45^{\circ}-\mathrm{Cl}$ unit-cell with a resulting saturation coverage of $\theta=0.5 \mathrm{ML}$ (normalized to the topmost substrate layer and corresponding to an absolute coverage of $\Gamma=7.7 \times 10^{14} / \mathrm{cm}^{2}$ ). In the STM approach such a structural correlation between the chloride adlayer and the underlying substrate is achieved by a systematic variation of the tunneling parameters (Fig. 2b-d). ${ }^{[16]}$ Chloride adsorption sites are exclusively fourfold-hollows (Fig. 2e). Not only is the atomic scale structure of the electrode surface affected by the chloride adsorption but also the entire surface morphology (Fig. $2 \mathrm{f}$ ), provided an adequate surface mobility has been induced at high electrode potentials. Chloride-stabilized substrate steps are aligned parallel to the $\langle 100\rangle$ substrate directions. These 'morphological effects' result from tremendous changes of the step and kink energies induced by the chloride adlayer. This morphological feature can be used as a 'fingerprint' for the presence of an ordered $\mathrm{c}(2 \times 2)-\mathrm{Cl}$ adlayer on $\mathrm{Cu}(100)$ even if the chloride layer is covered by an cationic organic film.

It is the order-disorder phase transition within the chloride adlayer at low poten-
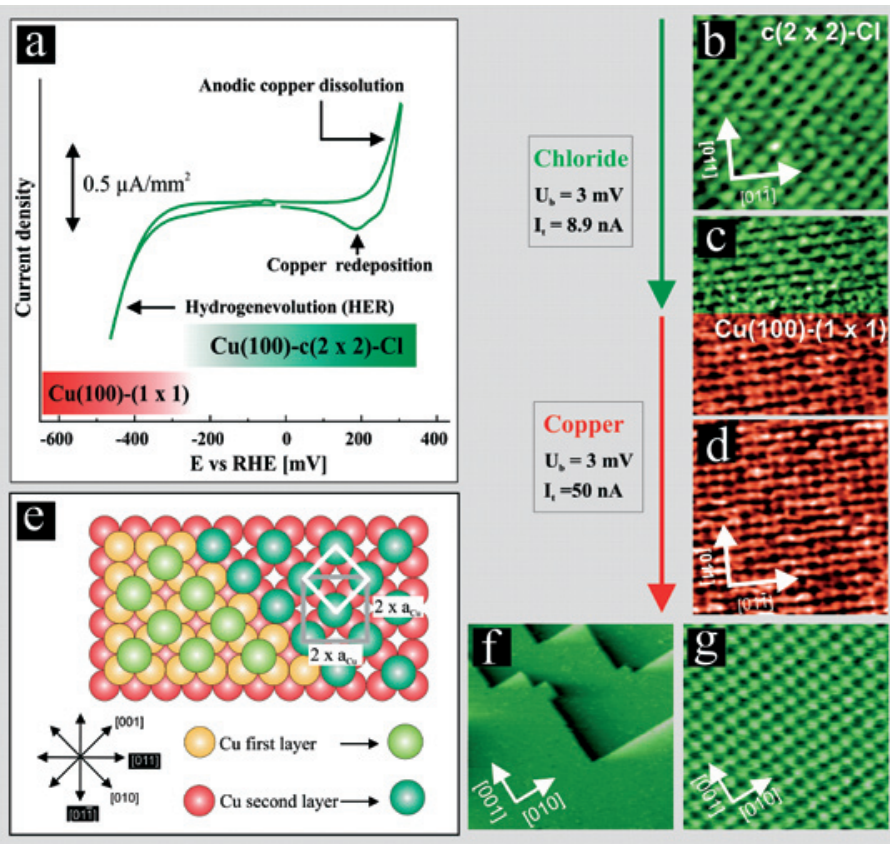

Fig. 2. a) Cyclic voltammogram of $\mathrm{Cu}(100)$ in $10 \mathrm{mM} \mathrm{HCl}$ indicating the stability regime of the laterally ordered chloride adlayer on $\mathrm{Cu}(100)$; b) - d) Correlation of the chloride adlayer to the underlying copper substrate by varying the tunneling parameters; e) hard-sphere model of the $\mathrm{c}(2 \times 2)-\mathrm{Cl}$ adlayer on $\mathrm{Cu}(100)$; f) Surface morphology in the presence of the ordered chloride adlayer, 69 $\mathrm{nm} \times 69 \mathrm{~nm}, \mathrm{I}_{\mathrm{t}}=1$ $\mathrm{nA}, \mathrm{U}_{\mathrm{b}}=25 \mathrm{mV}, \mathrm{E}_{\text {wo }}$ $=+100 \mathrm{mV}$ vs RHE; g) Atomic structure of the chloride saturation layer on $\mathrm{Cu}(100), 5.3$ $\mathrm{nm} \times 5.3 \mathrm{~nm}, \mathrm{l}_{\mathrm{t}}=1$ $\mathrm{nA}, \mathrm{U}_{\mathrm{b}}=25 \mathrm{mV}, \mathrm{E}_{\mathrm{w}}$ $=+100 \mathrm{mV}$ vs RHE; h) - k) Morphological changes induced by the order-disorder transition within the chloride adlayer, $47 \mathrm{~nm} \times 47 \mathrm{~nm}$, $\mathrm{I}_{\mathrm{t}}=1.7 \mathrm{nA}, \mathrm{U}_{\mathrm{b}}=255 \mathrm{mV}$. tials due to the starting chloride desorption that leads to a loss of the step-alignment as indicated in Fig. $2 \mathrm{~h}-\mathrm{k}$. The stability regime of the ordered $\mathrm{c}(2 \times 2)-\mathrm{Cl}$ adlayer as determined by in situ STM is indicated in the CV of Fig. 2a.

The entire $3 D$ interfacial structure including surface relaxations phenomena (interlayer spacings parallel to the surface normal direction, Fig. 3a) can be solved by applying modern in situ SXRD techniques. Surface X-ray diffraction from an ideal 2D crystalline surface gives rise to rods of scattered intensity in the reciprocal space which are oriented parallel to the surface normal and fulfill the Bragg condition only within the surface plane (Fig. $3 b){ }^{[17]} X$-ray diffraction from a real semiinfinite crystalline substrate gives rise to a superposition of these surface related rods of scattering with the bulk-related 3D Bragg peaks in the reciprocal space (CTR: crystal truncation rods). Structural surfacesensitive information (e.g. lateral structure, interlayer separations, surface roughness etc.) are derived from such a measurement by analyzing the intensity distribution of scattered X-rays along these CTRs between the Bragg peaks. ${ }^{[17]}$ Additional rods of scattering appear in the reciprocal space when superstructures, e.g. due to adsorption-related adlayer formation phenomena, occur at the surface which, hence, structurally deviates from the ideal arrangement of substrate atoms parallel to the surface plane in the crystal interior (adlayer rods, Fig. 3b).

The basis for our structure determination of the $\mathrm{Cu}(100) / \mathrm{Cl}$ system were the (1 1 L) and (0 2 L) Cu CTRs (Fig. 3c).

At $\mathrm{E}_{\text {work }}=0 \mathrm{mV}$ we find $\mathrm{a} \mathrm{Cu}-\mathrm{Cu}$ interlayer separation of $\mathrm{d}_{1-2}=1.846 \AA$ that is expanded by $2.2 \%$ with respect to the corresponding interlayer spacing in the $\mathrm{Cu}$ bulk material parallel to the surface normal with $\mathrm{d}_{\mathrm{Cu}-\mathrm{Cu}}=1.810 \AA$. Note that the clean $\mathrm{Cu}(100)$ surface under UHV conditions experiences a $1 \%$ contraction with $\mathrm{d}_{1-2}=$ $1.792 \AA .{ }^{[18]}$ Two effects are considered as the origin of such an 'anomalous' huge interlayer expansion of the topmost copper layers in the electrochemical environment. It can be assumed that the applied potentials are already above the $p z c$ (point of zero charge) thus involving a depletion of electrons at the electrode surface which in turn also affects the structural properties of the topmost $\mathrm{Cu}$ layers. In a simplified picture such a 'positive electrode polarization' causes the positive charge of the $\mathrm{Cu}$ cores to be less shielded by the electrons thus leading to an increased layer separation between the topmost copper layers due to electrostatic repulsion (potential/ charging effect on $\mathrm{d}_{12}$ ). Moreover it can be assumed that the adsorption of an anionic species that largely retains its negative charge upon adsorption even at these high 


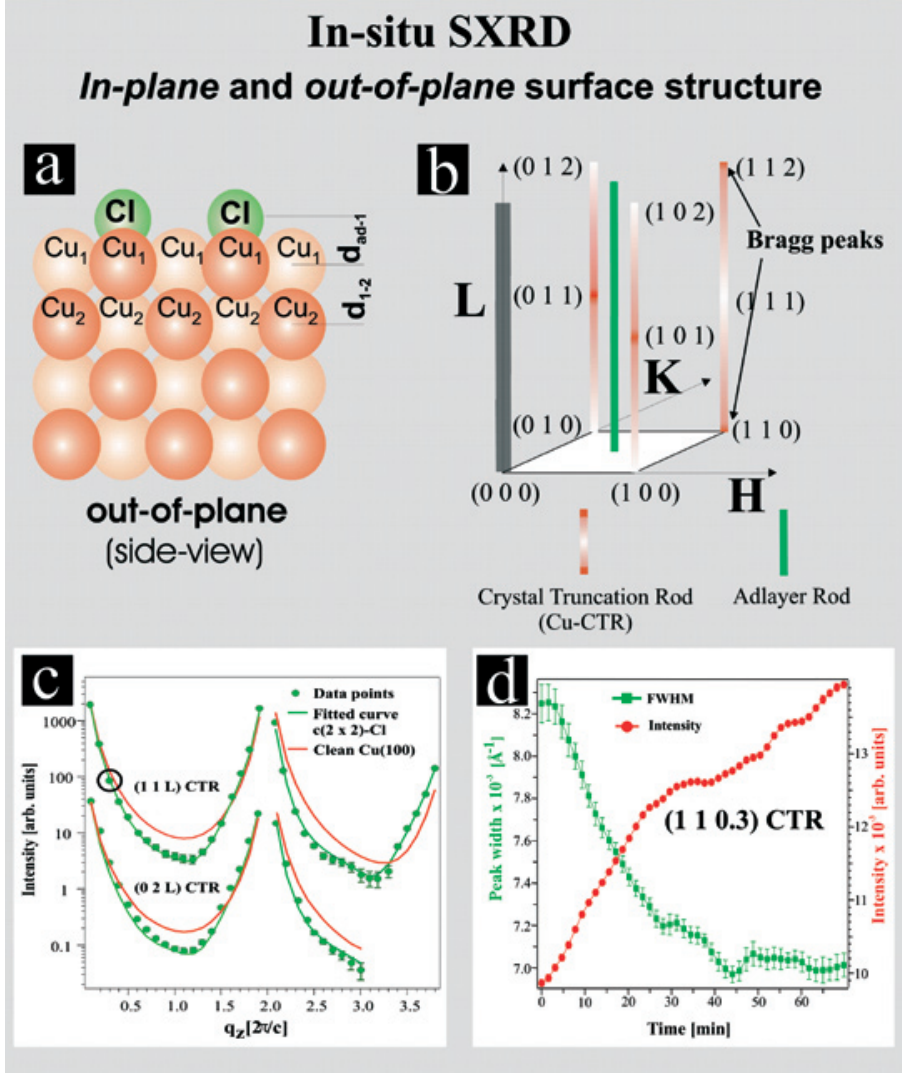

Fig. 3. In situ SXRD results revealing the entire three-dimensional structure of the electrified interface in the presence of the anion layer. (The X-ray scattering experiments reported here were carried out under potential control in an electrochemical cell at beamline ID32 at the European Synchrotron Radiation Facility ESRF in Grenoble $\left.{ }^{[16]}\right)$ : a) Schematic drawing indicating the out-of plane structure of the anionmodified electrode; b) Schematic drawing illustrating the surface X-ray diffraction from the halide modified fcc(100) surface in reciprocal space coordinates; c) Experimentally resolved Cu crystal truncation rods (CTRs) from the chloride-modified electrode surface obtained at $\mathrm{E}_{\text {work }}$ $=+100 \mathrm{mV}$; d) Chloride-mediated annealing of the copper surface monitored by in situ SXRD.

potentials should additionally increase such an interlayer expansion due to a further depletion of electrons which originates from the adsorbate-induced 'depolarization' of the substrate and related to that to an 'image charge effect' as discussed by Koper ${ }^{[19]}$ (adsorption effect on $\mathrm{d}_{12}$ ). The observed expanded $\mathrm{Cu}-\mathrm{Cu}$ interlayer separation in the presence of chloride indicates a considerable weakening of the $\mathrm{Cu}-\mathrm{Cu}$ bond at the surface. This might be a further reason explaining the experimentally observed rapid 'electrochemical annealing' of the copper surface in the presence of chloride (Fig. 3d) which has been so far ascribed exclusively to the excellent capabilities of chloride anions to complex cupric and cuprous ions and not to an additional adsorbate-triggered weakening of the $\mathrm{Cu}-\mathrm{Cu}$ bond.

The assumption of an adsorbed chloride anion that retains almost its full negative charge on $\mathrm{Cu}(100)$ within the ordered adlayer becomes further supported by the detected $\mathrm{Cu}$-halide interlayer separation $\mathrm{d}_{\mathrm{ad}-1}$ of $d_{\text {ad-1 }}=1.914 \AA$. Respective values of the
$\mathrm{Cu}-\mathrm{Cl}$ interlayer spacing observed for the chlorine adlayer on $\mathrm{Cu}(100)$ produced by a dissociative halogen adsorption in UHV are up to $20 \%$ smaller than the value found for the electrochemical environment. ${ }^{[20]}$ This huge difference has been attributed to a solvation effect by water dipoles and counter ions that accumulate in the electrochemical environment on top of the layer of specifically adsorbed chloride anions. ${ }^{[20]}$ Their presence reduces the charge transfer from the chloride anion to the metal since the energy gain by such a solvation effect is higher when the adsorbed anion remains charged. ${ }^{[19,21]}$ The enormous impact of the water dipoles and counter ions present in the outer Helmholtz layer on the particular charge and binding state of specifically adsorbed halides in the inner Helmholtz layer has recently been confirmed and modelled by quantum-mechanical DFT calculations in Bredow's group. [21]

It should be noted that no significant changes of the interlayer spacings were observed for potentials within the double lay- er regime quite in contrast to copper that has been modified by bromide anions. ${ }^{[21]}$ This significant difference has been attributed to the better polarizability of the adsorbed bromide that can be charged and discharged by potential changes thus impacting the interlayer spacings. ${ }^{[21]}$

The particular charge state of the specifically adsorbed anion is assumed to be important for the subsequent adsorption of cationic organic species (e.g. levelers in the course of the copper plating) in particular when electrostatic attraction is the main driving force for the adsorption process. ${ }^{[13]}$

\subsection{Anion-Cation Interaction at the Interface}

Fig. 4a depicts the molecular structure and dimension of the 1,1'-diphenyl-4,4'bipyridinium molecule (DPV) used for the adsorption experiments reported here. Note that the bipyridinium moiety of the viologen molecule undergoes significant intramolecular changes upon electron transfer ${ }^{[12]}$ as recently demonstrated by quantum-chemical DFT calculations for the 1,1'-dibenzyl-4,4'-bipyridinium molecule (DBV). ${ }^{[13 b]}$ The di-cationic viologen species typically exhibits a non-zero interplanar dihedral-angle $\Phi$ between the two pyridinium rings $\left(\right.$ e.g. $\left.\Phi\left(\mathrm{DBV}^{2+}\right)=40.1^{\circ}\right)$, at least in the absence of any external forces. However, a decrease of the dihedral-angle $\Phi$ is commonly observed upon reduction of the isolated di-cation to the corresponding radical mono-cation with a resulting dihedral-angle that is close to zero (e.g. $\left.\Phi\left(\mathrm{DBV}^{\bullet+}\right)=-5.4^{\circ}\right) .^{[13 \mathrm{~b}]}$ This transition can be seen as a crucial pre-requisite for the formation of stacked assemblies as the characteristic structural motif for violo-
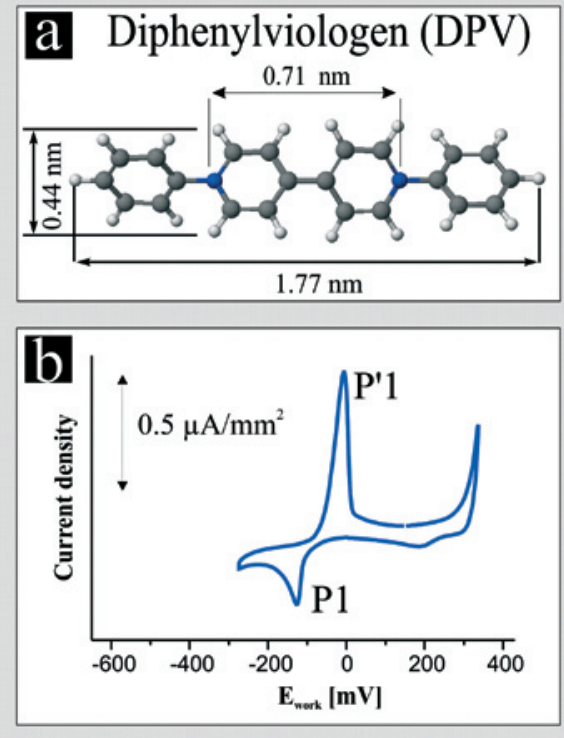

Fig. 4. a) Molecular structure of the 1,1'-4,4'-diphenyl-bipyridinium di-cation (diphenyl-viologen, abbreviated as DPV); b) CV of $\mathrm{Cu}(100)$ in $10 \mathrm{mM} \mathrm{HCl}+0.1 \mathrm{mM} \mathrm{DPVCl}_{2}$. 


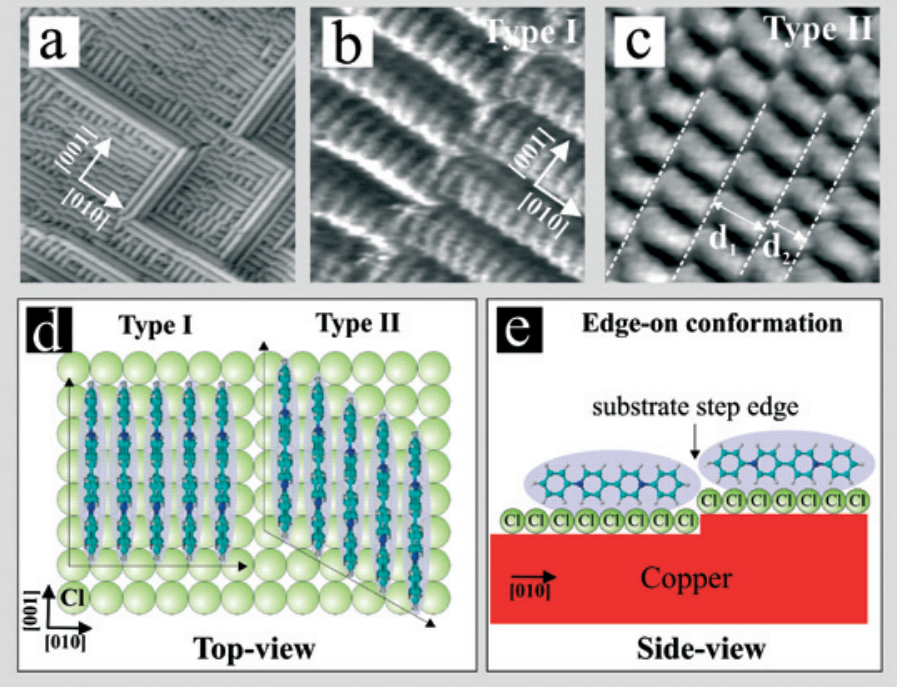

Fig. 5. Surface morphology in the presence of the poly-cationic $\left(\mathrm{DPV}^{+}\right)_{\mathrm{n}}$ stacking phase on the chloride lattice, a) $57.6 \mathrm{~nm} \times 57.6 \mathrm{~nm}, \mathrm{I}_{\mathrm{t}}=0.6 \mathrm{nA}$, $\left.\mathrm{U}_{\mathrm{b}}=250 \mathrm{mV}, \mathrm{E}_{\text {work }}=0 \mathrm{mV}, \mathrm{b}\right)$ Type-I $\left(\mathrm{DPV}_{a d s}^{*+}\right)_{\mathrm{n}}$ stacking assembly, 5.7 $\mathrm{nm} \times 5.7 \mathrm{~nm}, \mathrm{I}_{\mathrm{t}}=0.5 \mathrm{nA}, \mathrm{U}_{\mathrm{b}}=270 \mathrm{mV}, \mathrm{E}_{\text {work }}=0 \mathrm{mV}$; c) Type-II $\left(\mathrm{DPV}_{a d s}^{*+}\right)_{\mathrm{n}}$ stacking assembly, $6.7 \mathrm{~nm} \times 6.7 \mathrm{~nm}, \mathrm{I}_{\mathrm{t}}=0.6 \mathrm{nA}, \mathrm{U}_{\mathrm{b}}=250 \mathrm{mV}, \mathrm{E}_{\text {work }}=0$ $\mathrm{mV}$; d) In-plane structural model of the $\left(\mathrm{DPV}_{a d s}^{*+}\right)_{\mathrm{n}}$ stacking phases on the chloride lattice; e) Out-of-plane structural model of the $\left(\mathrm{DPV}_{a d s}^{*+}\right)_{\mathrm{n}}$ stacking phases on the chloride lattice.

gens in their radical mono-cationic state at the electrode surface. ${ }^{[13 a]}$

Changes in the electrochemical potential/current response of the system due to the presence of the $\mathrm{DPV}^{2+}$ in solution are presented in Fig. 4b. Peak system P1/P'1 has to be attributed to the first DPV reduction step (Eqn. 1). ${ }^{[22]}$ The extraordinary reactivity of the $\mathrm{DPV}^{2+}$ can be concluded from a comparison with electrochemical data of the $\mathrm{DBV}^{2+}$ reduction that is shifted by $\Delta \mathrm{E}_{\mathrm{w}}$ $=-256 \mathrm{mV}$ to lower potentials. The DPV obviously stabilizes the radical much more effective than the less reactive DBV. ${ }^{[13]}$

Fig. 5 provides an overview about the 2D structures of the poly-cationic DPV film adsorbed on the $\mathrm{c}(2 \times 2)-\mathrm{Cl}$ adlattice on the $\mathrm{Cu}(100)$ surface within the potential regime above $\mathrm{P} 1$ where the DPV is expected to be in its di-cationic state, at least in the solution phase. The presence of a structurally intact $\mathrm{c}(2 \times 2)-\mathrm{Cl}$ phase under the organic layer can be concluded from the preferential substrate step-alignment along the $\langle 100\rangle$ directions (Fig. 5a).

Characteristic structural motifs of the DPV adlayer are stacked assemblies, even at these high potentials. This observation appears surprising since this stacking motif has been identified as a structural 'fingerprint' for the presence of mono-reduced viologen species but not for the presence of adsorbed viologens in their di-cationic redox-state. ${ }^{[13 a]}$ This experimental result has been interpreted by a DPV species that cannot be stabilized in its di-cationic form on the copper surface at any potential within the narrow potential window of copper. Instead it is assumed to react instantaneously to the corresponding monoreduced species even at potentials above the main redox-wave of the respective solution species. A similar phenomenon has been reported by Sagara's group for the adsorption of various viologens on HOPG and $\mathrm{Hg}$ surfaces. ${ }^{[23]}$

$\left(\mathrm{DPV}_{a d s}^{*+}\right)_{\mathrm{n}}$ stacked chains right after the reactive DPV adsorption (Fig. 5a) are preferentially aligned parallel to the substrate $\langle 100\rangle$ directions with individual $\mathrm{DPV}_{a d s}^{*+}$ molecules oriented with their main molecular $\mathrm{N}-\mathrm{N}$-axis perpendicular to the respective step edge (parallel to the other $\langle 100\rangle$ directions). The NND (nearest neighbor distance) of $\mathrm{DPV}_{a d s}^{\circ+}$ entities within these stacked chains amounts to $\mathrm{NND}_{\mathrm{DPV}}=0.36(2) \mathrm{nm}$ which is identical to the NND of the underlying chloride lattice of $\mathrm{NND}_{\mathrm{c}(2 \times 2)-\mathrm{Cl}}=0.362 \mathrm{~nm}$ and identical to the intermolecular spacings of monoreduced $\mathrm{DBV}_{a d s}^{*+}$ species on the $\mathrm{c}(2 \times 2)-\mathrm{Cl} /$ $\mathrm{Cu}(100){ }^{[13 b]}$ This observation points to a certain 'coordinative' effect of the chloride lattice on the covering film of $\mathrm{V}^{\bullet+}$ species although intermolecular $\pi-\pi$-interactions are assumed to dominated the phase behavior of viologens in their mono-reduced state while electrostatic interactions are discussed as more important for the nonreactive adsorption of viologens on the chloride-modified electrode surface. ${ }^{[13]}$ Note this 'coordinative' effect of the chloride lattice on the covering $\mathrm{DPV}_{\text {ads }}^{*+}$ film is less pronounced on the $\mathrm{c}(2 \times 2)-\mathrm{Br} / \mathrm{Cu}(100)$ where the bromide is largely discharged at high potentials. ${ }^{[24]}$
We propose a structure model that assumes $\mathrm{DPV}_{a d s}^{++}$entities adsorbed on the $\mathrm{c}(2$ $\times 2$ )-Cl lattice in so-called 'edge-on' geometry with the molecular N-N-axes parallel to the surface (Fig. 5d). The inter-chain distance amounts to $\mathrm{d}=1.88(2) \mathrm{nm}$ and compares well with the length of the DPV molecules (Fig. 4a). An interdigitation of the phenyl groups of $\mathrm{DPV}_{a d s}^{\circ+}$ entities into adjacent $\left(\mathrm{DPV}_{a d s}^{*+}\right)_{\mathrm{n}}$ chains can therefore be ruled out. It should be noted that the $\left(\mathrm{DPV}_{a d s}^{*+}\right)_{\mathrm{n}}$ phase observed right after the initial adsorption (type-I phase) undergoes a slow 'ripening' process leaving an altered and energetically more favorable stacking motif behind (type-II, Fig. 5c) where individual $\mathrm{DPV}_{a d s}^{*+}$ entities are still oriented with their main molecular axis parallel to the substrate $\langle 100\rangle$ directions but with neighbored $\mathrm{DPV}_{a d s}^{\circ+}$ entities that are slightly shifted with respect to each other (type-II stacking). Within this stacked phase $\mathrm{DPV}_{a d s}^{*+}$ molecules self-assemble into several kinds of oligomers, e.g. ( $\left.\mathrm{DPV}_{a d s}^{*+}\right)_{6}$ hexamers and $\left(\mathrm{DPV}_{a d s}^{\cdot+}\right)_{4}$ tetramers that order themselves laterally on the chloride lattice (Fig. 5c).

It is worth mentioning that the lateral order of the $\left(\mathrm{DPV}_{a d s}^{*+}\right)_{\mathrm{n}}$ stacked phase is particularly well developed at the monoatomically high substrate steps. This results in two almost defect-free stacked chains on either side of these step edges, i.e. the upper and the lower side (Fig. 5a). This structural feature has to be regarded as the origin for the inhibiting effect of this particular anion-cation layer on the copper dissolution and deposition reaction. ${ }^{[24]}$ Substrate step edges as the reactive sites for deposition and dissolution processes are effectively blocked by the $\mathrm{DPV}_{a d s}^{\cdot+}$. [24]

Information on the chemical composition of the paired anion-cation layer on $\mathrm{Cu}(100)$ can be obtained by ex situ photoemission experiments (Fig. 6). Of particular interest is here the chemical shift of the N1s emission which is sensitive to the redox-state of the adsorbed viologen species. The dominant N1s emission at $\mathrm{BE}=$ 400.71(2) in Fig. 6 points to the presence of reduced $\mathrm{DPV}_{a d s}^{\cdot+}$ species at the electrode surface although $\mathrm{DPV}_{a q}^{2+}$ solution species are stable in their di-cationic form at these high electrode potentials. Our assignment of the main N1s peak in Fig. 6 to the monoreduced $\mathrm{DPV}_{a d s}^{\circ+}$ species is clearly supported by measurements on the N1s binding energy of the di-cationic $\mathrm{DPV}^{2+}$ species in the solid $\mathrm{DPVCl}_{2}$ salt. The latter was also deposited on an inert Au foil. As expected, we found di-cationic $\mathrm{DPV}^{2+}$ at high binding energies of $\mathrm{BE}=402.53$ (2) $\mathrm{eV}$ (see XPS spectrum (1) in Fig. 6) as the main component. However, $\mathrm{DPV}^{2+}$ in the respective $\mathrm{DPVCl}_{2}$ salt suffers reduction upon X-ray irradiation. In line with the disappearance of the di-cationic DPV ${ }^{2+}$ species a broad satellite feature emerges with time in the 


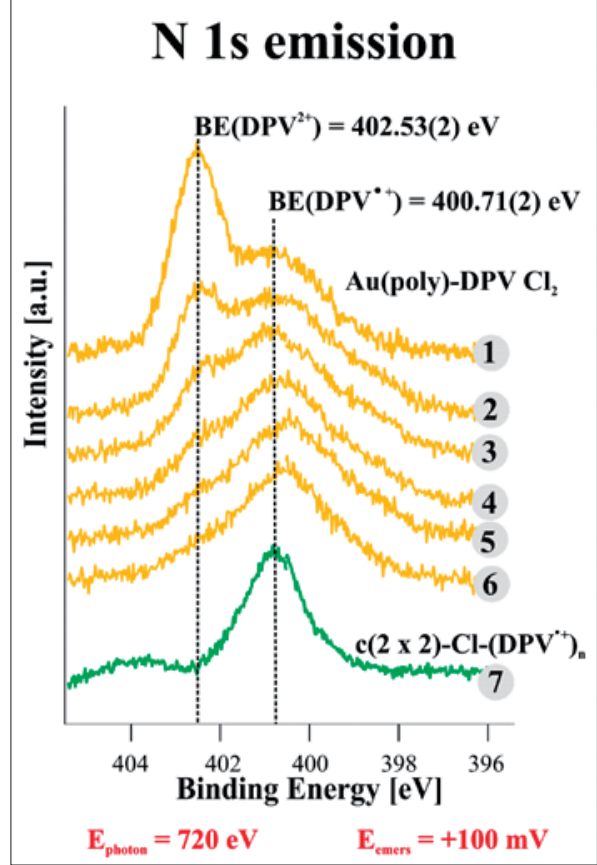

Fig. 6. Ex situ XPS data: Time-dependent $\mathrm{N} 1 s$ photoemission spectra of the $\mathrm{DPVCl}_{2}$ salt deposited on an inert gold foil (internal reference) compared with the N1s photoemission spectrum obtained from the $\left(\mathrm{DPV}_{a d s}^{++}\right)_{\mathrm{n}}$ stacking phase on $\mathrm{c}(2 \times 2)-\mathrm{Cl}$ at high potentials, $\mathrm{E}_{\text {photon }}=720 \mathrm{eV}$. (Photoemission studies were performed using the SoLiAS (Solid/Liquid interface Analysis System) experimental station at the undulator beamline U49/2-PGM2 at the synchrotron light source BESSY II. Electrochemical processing was performed in an atmospheric pressure inertgas cell using a classical hanging meniscus configuration followed by a transfer of the electrode surface into the vacuum via a purpose-designed load lock. ${ }^{[22]}$ )

N1s photoemission spectrum also around the value as in the adsorbed case, i.e. $\mathrm{BE}$ $=400.71(2) \mathrm{eV}$, representing reduced DPV species as reaction products of the 'dry' $\mathrm{DPV}^{2+}$ reduction caused by slow secondary electrons and Auger electrons. The emergence of this reduced DPV ${ }^{\bullet+}$ species in the $\mathrm{DPVCl}_{2}$ salt upon radiation again serves as reference for the spectroscopic identification of the mono-reduced $\mathrm{DPV}_{a d s}^{++}$species on the halide-modified copper surface.

These results become further supported by potential-dependent STM measurements presented in Fig. 7. While the di-cationic $\mathrm{DBV}_{\text {ads }}^{2+}$ phase undergoes a distinct phase transition upon passing the first redox-wave in a negative-going potential sweep in the CV as reported by Pham et $a l .{ }^{[13 \mathrm{~b}]}$ there are no structural transitions observed within the $\left(\mathrm{DPV}_{a d s}^{*+}\right)_{\mathrm{n}}$ phase when the main reduction peak $\mathrm{P} 1$ is passed. This finding can simply be explained by assuming adsorbed DPV species that are already reduced before reaching $\mathrm{P} 1$ in the $\mathrm{CV}$ (Fig. $4 \mathrm{~b})$. Note that the anion-cation layer remains structurally stable when potentials
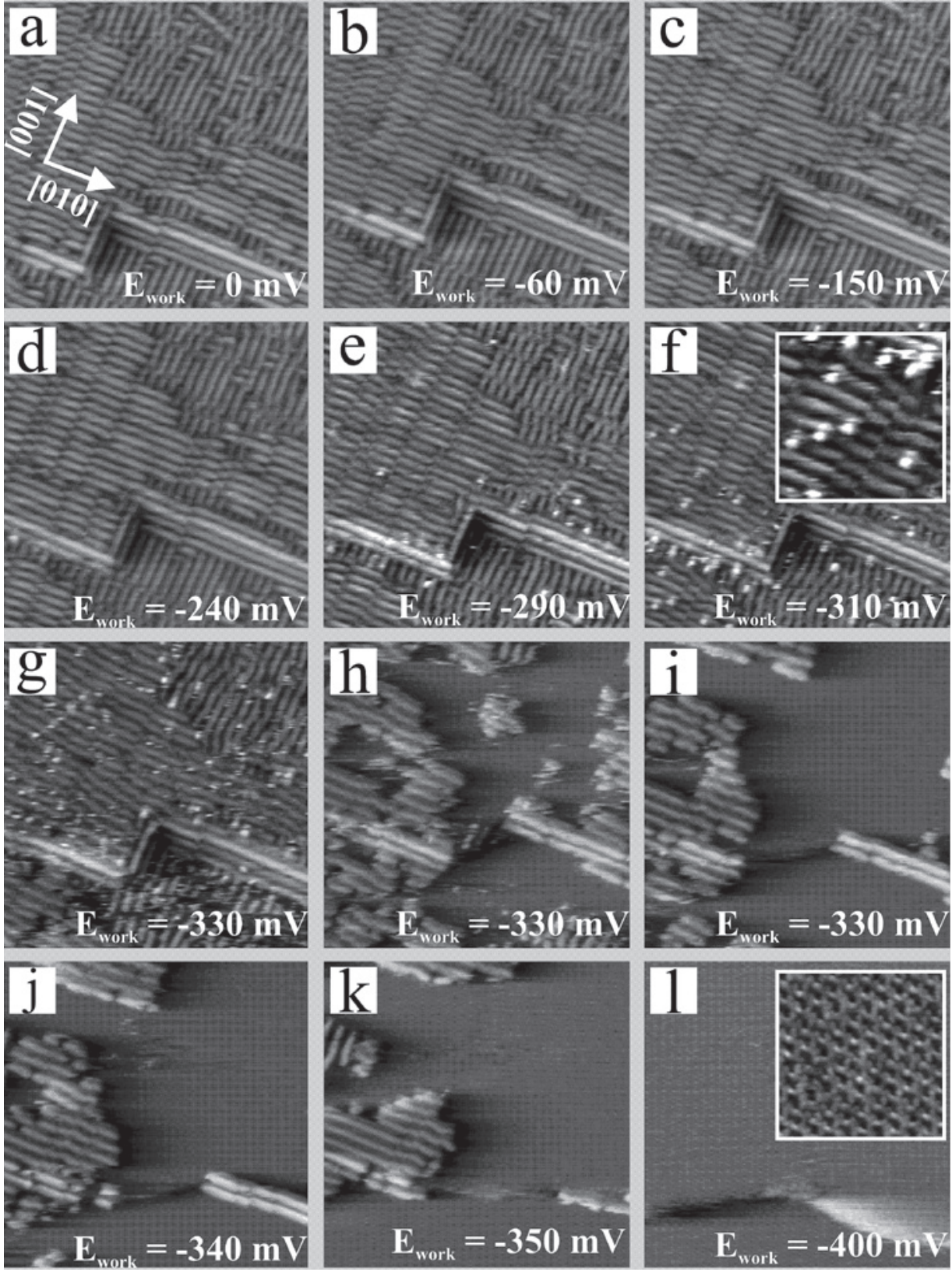

Fig. 7. Potential-dependent series of STM images demonstrating the decay of the $\left(\mathrm{DPV}_{a d s}^{*+}\right)_{\mathrm{n}}$ stacking phase induced by the on-set of chloride desorption through the poly-cationic organic layer, $50 \mathrm{~nm} \times 50 \mathrm{~nm}, \mathrm{I}_{\mathrm{t}}=0.2 \mathrm{nA}, \mathrm{U}_{\mathrm{b}}=300 \mathrm{mV}$.

are applied where the massive reduction of $\mathrm{DPV}^{2+}$ solution species sets in.

The decay of the $\left(\mathrm{DPV}_{a d s}^{*}\right)_{\mathrm{n}}$ stacking at potentials below $\mathrm{E}_{\text {work }}=-300 \mathrm{mV}$ has to be attributed to the starting chloride desorption through the $\left(\mathrm{DPV}_{a d s}^{*+}\right)_{\mathrm{n}}$ adlayer as can clearly be concluded from the loss of the preferential alignment of substrate steps along the $\langle 100\rangle$ directions (Fig. 7h-1). $\mathrm{DPV}_{\text {ads }}^{++}$species change their adsorption geometry at these low potentials from edgeon to side-on (Fig. 1) thus facilitating the interaction of the radical cations with metallic copper surface. Whether a certain amount of chloride anions are still co-adsorbed within this layer (Fig. 1c) cannot be concluded or excluded on the basis of the available STM data.
Compared to the DPV-free electrolyte the order-disorder transition within the chloride adlayer is shifted to lower potentials by about $100 \mathrm{mV}$ due to the presence of the covering $\left(\mathrm{DPV}_{a d s}^{*+}\right)_{\mathrm{n}}$ stacked layer. A similar phenomenon has recently been reported for $\left(\mathrm{DBV}_{a d s}^{*+}\right)_{n}$ layers on the chloride modified copper surface. ${ }^{[13 b]}$

Chloride re-adsorption through the $\mathrm{DPV}_{a d s}^{*+}$ layer on the bare metallic copper leads to the full restoration of the paired anion-cation layer (Fig. 8). The penetration of the chloride anions through the organic film locally starts at domain boundaries as indicated by the black dotted lines in Fig 8a. 

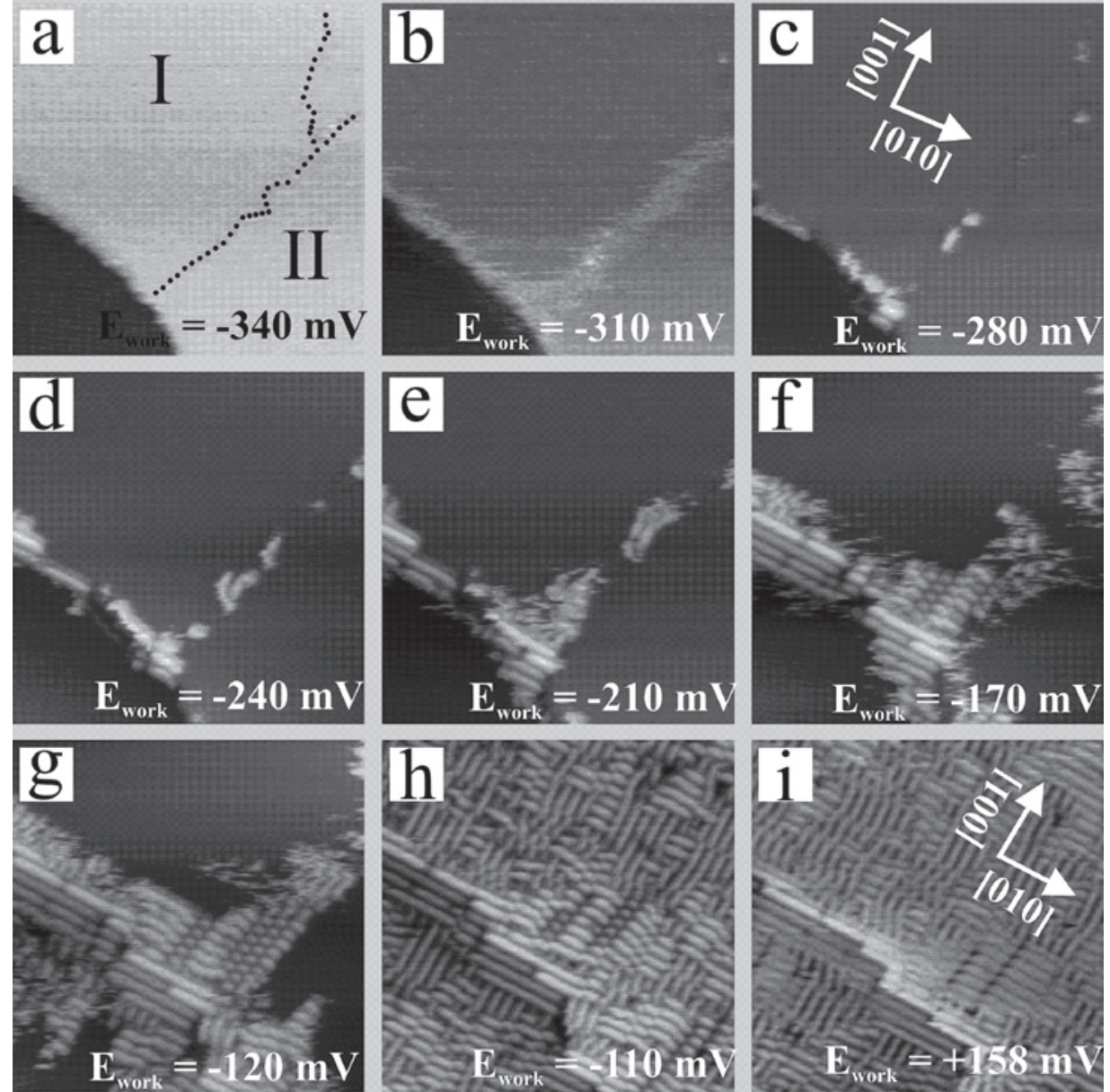

Fig. 8. Potential-dependent series of STM images demonstrating the formation of the $\left(\mathrm{DPV}_{a d s}^{*+}\right)_{\mathrm{n}}$ stacking phase induced by the chloride re-adsorption through the poly-cationic organic layer, $50 \mathrm{~nm} \times 50 \mathrm{~nm}, \mathrm{I}_{\mathrm{t}}=0.2 \mathrm{nA}, \mathrm{U}_{\mathrm{b}}=300 \mathrm{mV}$.

\section{Conclusions}

Diphenyl-viologens (DPV) can be regarded as prototype for a highly reactive viologen species. It cannot be adsorbed and stabilized in its oxidized di-cationic form on the chloride modified electrode surface, at least within the narrow potential window of copper. Quite intriguing, DPV ${ }^{2+}$ instantaneously reacts in the presence of an ordered halide layer to the corresponding radical mono-cation $\mathrm{DPV}_{a d s}^{*+}$ at the chloridemodified surface followed by the formation of a condensed ( $\left.\mathrm{DPV}_{a d s}^{*+}\right)_{\mathrm{n}} \pi$-stacked 'stripe' phase at potentials more positive than the main redox-wave in the $\mathrm{CV}$. This stacked layer has to be considered as hydrophobic which completely removes all water species from the chloride lattice. Upon adsorption and phase formation the DPV releases almost completely its solvation shell as a pre-requisite to undergo strong inter-viologen interactions, most likely in the form of $\pi-\pi$-interactions. Strong adsorbate-adsorbate interactions are considered as the main driving forces for the $\left(\mathrm{DPV}_{a d s}^{*+}\right)_{\mathrm{n}}$ phase formation although a certain 'coordinative' effect by the underlying chloride orients individual $\mathrm{DPV}_{a d s}^{*+}$ entities along the substrate $\langle 100\rangle$ directions. (Quasi)revers- ible chloride desorption through the organic layer leads to a reorientation of the $\mathrm{DPV}_{a d s}^{*+}$ entities from an edge-on to a sideon adsorption geometry.

\section{Received: January 15, 2009}

[1] M. F. Toney, J. N. Howard, J. Richer, G. L. Borges, J. G. Gordon, O. R. Melroy, D. G. Wiesler, D. Yee, L. B. Sorensen, Surf. Sci. 1995, 335, 326.

[2] a) P. Lustenberger, H. Rohrer, R. Christoph, H. Siegenthaler, J. Electroanal. Chem. 1988, 243, 225; b) K. Itaya, E. Tomita, Surf. Sci. 1988, 201, L507; c) J. Wiechers, T. Twomey, D. M. Kolb, R. J. Behm, J. Electroanal. Chem. 1988, 248, 451.

[3] P. C. Andricacos, C. Uzoh, J. O. Dukovic, J. Horkans, H. Deligianni, IBM J. Res. Dev. 1998, 42,567

[4] Q. Huang, B. C. Maker-O'Neal. J. J. Kelly, P. Broekmann, A. Wirth, M. Martin, M. Hahn, A. Wagner, D. Mayer, Electrochem. Solid-State Lett., accepted for publication.

[5] a) E. D. Eliadis, R. G. Nuzzo, A. A. Gewirth, R. C. Alkire, J. Electrochem. Soc. 1997, 144, 96; b) M. Hasegawa, Y. Negishi, T. Nakanishi, T. Osaka, J. Electrochem. Soc. 2005, 152, C221; c) M. Tan, C. Guymon, D. R. Wheeler, J. N. Harb, J. Electrochem. Soc. 2007, 154, D78; d) P. M. Vereecken, R. A. Binstead, H. Deligianni, P. C. Andricacos, IBM J. Res. Dev. 2005, 49, 3.

[6] a) J. J. Kelly, A. C. West, J. Electrochem. Soc. 1998, 145, 3472; b) J. J. Kelly, A. C. West, $J$. Electrochem. Soc. 1998, 145, 3477.

[7] a) D. Josell, D. Wheeler, W. H. Huber, T. P. Moffat, Phys. Rev. Lett. 2001, 8701; b) D. Josell,
D. Wheeler, T. P. Moffat, Electrochem. Solid-State Lett. 2002, 5, C49; c) T. P. Moffat, D. Wheeler, W. H. Huber, D. Josell, Electrochem. Solid-State Lett. 2001, 4, L5; d) D. Josell, D. Wheeler, W. H. Huber, J. E. Bonevich, T. P. Moffat, J. Electrochem. Soc. 2001, 148, C767; e) T. P. Moffat, D. Wheeler, W. H. Huber, D. Josell, Electrochem. Solid-State Lett. 2001, 4, C26; f) T. P. Moffat, D. Wheeler, S. K. Kim, D. Josell, J. Electrochem. Soc. 2006, 153, C127; g) T. P. Moffat, D. Wheeler, C. Witt, D. Josell, Electrochem. Solid-State Lett. 2002, 5, C110.

[8] a) K. Doblhofer, S. Wasle, D. M. Soares, K. G. Weil, G. Weinberg, G. Ertl, Zeit. Phys. Chem. 2003, 217, 479; b) K. Doblhofer, S. Wasle, D. M. Soares, K. G. Weil, G. Ertl, J. Electrochem. Soc. 2003, 150, C657.

[9] D. Wang, R. D. Mikkola, C. Wu, G. G. Barclay, US patent US20060016693A1.

[10] S. K. Kim, D. Josell, T. P. Moffat, J. Electrochem. Soc. 2006, 153, C616.

[11] a) D. W. Suggs, A. J. Bard, J. Phys. Chem. 1995 99, 8349; b) R. Vogt, F. A. Möller, C. M. Schilz, O. M. Magnussen, R. J. Behm, Surf. Sci. Lett. 1996, 367, L33; c) R. Vogt, A. Lachenwitzer, O. M. Magnussen, R. J. Behm, Surf. Sci. 1998, 399, 49; d) O. M. Magnussen, M. R. Vogt, Phys. Rev. Lett. 2000, 85, 357; e) O. M. Magnussen, L. Zitzler, B. Gleich, M. R. Vogt, R. J. Behm, Electrochim. Acta 2001, 46, 3725.

[12] a) C. L. Bird, A. T. Kuhn, Chem. Soc. Rev. 198?, 110, 49; b) P. M. S. Monk, 'The Viologens: Physicochemical Properties, Synthesis and Applications of the Salts of 4,4'-Bipyridines', John Wiley \& Sons Ltd., Chichester, England, 1989.

[13] a) D. T. Pham, S.-L. Tsay, K. Gentz, C. Zoerlein, S. Kossmann, J.-S. Tsay, B. Kirchner, K. Wandelt, P. Broekmann, J. Phys. Chem. C 2007, 111, 16428; b) D. T. Pham, K. Gentz, C. Zoerlein, N. T. M. Hai, S. L. Tsay, B. Kirchner, S. Kossmann, K. Wandelt, P. Broekmann, N. J. Chem. 2006, 30, 1439 ; c) C. Safarowsky, A. Rang, C. A. Schalley, K. Wandelt, P. Broekmann, Electrochim. Acta 2005, 50, 4257 .

[14] O. M. Magnussen, Chem. Rev. 2002, 102, 679.

[15] A. N. Frumkin, Z. Physik. Chem. 1933, A164, 121.

[16] S. Huemann, N. T. M. Hai, P. Broekmann, K. Wandelt, H. Zajonz, H. Dosch, F. Renner, J. Phys. Chem. B 2006, 110, 24955.

[17] I. K. Robinson, D. J. Tweet, Rep. Progr. Phys 1992, 55, 599

[18] a) M. A. Abu-Joudeh, B. M. Davies, P. A Montano Surf. Sci. 1986, 171, 331; b) D. M. Lind, F. B. Dunning, G. K. Walters, H. L. Davies, Phys. Rev. B 1987, 35, 9037.

[19] M. T. M. Koper, J. Electroanal. Chem. 1998, 450, 189.

[20] a) L. Q. Wang, A. E. Schach von Wittenau, Z. G. Ji, L. S. Wang, Z. Q. Huang, D. A. Shirley, Phys. Rev. B 1991, 44, 1292; b) P. H. Citrin, D. R. Hamann, L. F. Mattheiss, J. E. Rowe, Phys. Rev. Lett. 1982, 49, 1712; c) F. Jona, D. Westphal, A. Goldman, P. M. J. Marcus, Physics 1983, C16, 3001; d) J. R. Patel, D. W. Berreman, F. Sette, P. H. Citrin, J. E. Rowe, P. L. Cowan, T. Jach, B. Karlin, Phys. Rev. B 1989, 40, 1330.

[21] M. Saracino, M. Becker, H. Keller, H. Dosch, P. Broekmann, T. Bredow, K. Gentz, K. Wandelt, Phys. Rev. B, submitted.

[22] S. Breuer, D. T. Pham, S. Huemann, K. Gentz, C. Zoerlein, R. Hunger, K. Wandelt, P. Broekmann, N. J. Phys. 2008, 10, 1

[23] a) T. Sagara, K. Miuchi, J. Electroanal. Chem. 2004, 567, 193; b) T. Sagara, S. Tanaka, Y. Fukuoka, N. Nakashima, Langmuir 2001, 17, 1620 ; c) T. Sagara, S. Tanaka, K. Miuchi, N. Nakashima, J. Electroanal. Chem. 2002, 524, 68.

[24] K. Gentz, P. Broekmann, K. Wandelt, in preparation. 